\title{
Fauna de Muscidae (Diptera) em três localidades do Estado do Paraná, Brasil, capturada por armadilha Malaise ${ }^{1}$
}

\author{
Nise do Carmo Costacurta ${ }^{2}$ \\ Renato C. Marinoni ${ }^{3,4}$ \\ Claudio J. B. de Carvalho 3,5
}

\begin{abstract}
Muscidae (Diptera) fauna from three sites of Parana State, Brazil, captured with Malaise trap. During two years, from August 1986 to July 1988, the entomofauna of some preserved areas of Parana State, southern Brazil, was sampled in a project called "Levantamento da Fauna Entomológica no Estado do Paraná (PROFAUPAR)". Specimens of Muscidae (Diptera) were sorted out from the material collected using Malaise traps in three of the eight sites sampled (Colombo, Ponta Grossa and Guarapuava) in the first year (August 1986 to July 1987). A total of 7,014 specimens of Muscidae was captured and 91 species were identified. Neodexiopsis flavipalpis Albuquerque was the most abundant species in Ponta Grossa (672 specimens) and in Guarapuava (332 specimens). For Colombo, the most abundant species was Neodexiopsis vulgaris Couri \& Albuquerque (172 specimens). The highest richness of species and abundance were observed in Ponta Grossa (77 and 3,559 respectively). The total number of specimens and means values of capture were analyzed. Indices of diversity and evenness were used to discuss richness and dominance of species in each locality. Besides using ecological indices, species richness estimators were also used.
\end{abstract}

KEYwORDS. Alpha diversity; beta diversity; faunistic; seasonality; species richness estimators.

\section{INTRODUÇÃO}

Neste trabalho são estudados os Muscidae (Diptera), capturados em armadilha Malaise em três localidades, Colombo, Ponta Grossa e Guarapuava, dentre as oito localidades que serviram para estudos pelo Projeto "Levantamento da Fauna Entomológica no Estado do Paraná" (PROFAUPAR) (MARINONI $\&$ DutRa 1993). Durante o primeiro ano de coleta (agosto de 1986 a julho de 1987), os Diptera foram bem representados em armadilha Malaise, nas três localidades, correspondendo a $85 \%$ do total de insetos capturados (265.427/314.121). Os Muscidae capturados atingiram 2,6\% do total de Diptera (7.014/265.427).

Os Muscidae são encontrados em todas as regiões biogeográficas do mundo, com mais de 4.000 espécies. Na região Neotropical são conhecidos 87 gêneros e 796 espécies (CARvalHo et al. 1993). No Paraná, antes deste estudo, estavam registrados cerca de 30 gêneros e 74 espécies, aproximadamente $34,48 \%$ e $9,30 \%$ da fauna Neotropical conhecida, respectivamente.

\section{MATERIALE MÉTODOS}

Locais de coleta. O material para estudo foi obtido de coletas realizadas em três dos oito locais de amostragem do PROFAUPAR . Escolheu-se um ponto de coleta em cada um dos três planaltos paranaenses, dando preferência à localidade com maior número de exemplares de Diptera.

A) Primeiro Planalto. A armadilha foi instalada na EMBRAPA, Empresa Brasileira de Pesquisa Agropecuária, Colombo, junto à rodovia Curitiba-Adrianópolis, antiga Estrada da Ribeira, no Km 19 (BR-476). Altitude: aproximadamente 915 metros. Latitude: $25^{\circ} 20^{\prime} \mathrm{S}$; Longitude: $49^{\circ} 14^{\prime} \mathrm{W}$.

Classificação fitogeográfica, segundo VeLoso \& GóES (1982): Floresta Ombrófila Mista Montana. Situação florística atual, segundo Hatschbach (in MARINONI \& DUTRA 1993): "Típico erval do primeiro Planalto com erva-mate, pinheiro, vassourão, caapororocão etc. Mato muito destruído, tendo sido invadido pela bracatinga, caapororoca, etc".

B) Segundo Planalto. Coleta realizada no Parque Estadual

\footnotetext{
1. Contribuição $n^{\circ} 1374$ do Departamento de Zoologia, Universidade Federal do Paraná.

2. Hospital de Clínicas, Universidade Federal do Paraná. Rua Padre Camargo 280, 80060-240 Curitba-PR, Brasil.

Endereço eletrônico: nisecc@hotmail.com

3. Departamento de Zoologia, Universidade Federal do Paraná. Caixa Postal 19020, 81531-980 Curitba-PR, Brasil.

4. Bolsista CNPq. Endereço eletrônico: rcmari@ufpr.br

5. Bolsista CNPq. Endereço eletrônico: cjbcarva@ufpr.br
} 
de Vila Velha, junto à Rodovia do Café, BR 376, Km 83, Ponta Grossa, em área de preservação estadual, sob controle do Instituto Ambiental do Paraná. Altitude: aproximadamente 880 metros. Latitude: $25^{\circ} 14^{\prime} \mathrm{S}$; Longitude: $50^{\circ} 03^{\prime} \mathrm{W}$.

Classificação fitogeográfica, segundo Veloso \& Góes (1982): Floresta Ombrófila Mista Montana. Situação florística, segundo Hatschbach (in MARINONI \& DutRa 1993): "Capão natural de campo com presença do pinheiro, diversas canelas e imbuia. Pobre em epífitas. Sofreu ação do homem com retirada de madeiras de lei, principalmente canelas e imbuia".

C) Terceiro Planalto. O ponto de coleta localizou-se na Estância Santa Clara, Guarapuava, no vale do Rio Jordão, encravado numa região de campos limpos (Campos de Guarapuava). Área pertencente à Paranatur (Empresa Paranaense de Turismo), distante 12 quilometros da Rodovia 373 (Três Pinheiros-Pato Branco). Altitude: aproximadamente 740 metros. Latitude: $25^{\circ} 40^{\prime} \mathrm{S}$; Longitude: $52^{\circ} 01^{\prime} \mathrm{W}$.

Classificação fitogeográfica, segundo VeLOSO \& Góes (1982): Transição de Floresta Ombrófila Mista e Floresta Estacional Semidecidual. Situação florística, segundo Hatschbach (in MARINONI \& DUTRA 1993): "Situada na orla dos campos gerais do Terceiro Planalto, é uma típica transição dos capões de campo com a mata de galeria do rio Iguaçu, que sobe pelo rio Jordão. $\mathrm{Na}$ parte mais alta predomina o pinheiro, ao lado das leguminosas de grande porte, e diversas lauráceas. Em direção ao vale, diminui o pinheiro e aparecem os elementos de galeria. Bastante destruída a mata sofreu retirada de pinheiros e canelas, principalmente. Há, no entanto, algumas pequenas áreas de mata bem conservada".

Coleta e identificação do material. O material foi coletado em Colombo, Ponta Grossa e Guarapuava, através de armadilha Malaise (Townes 1972). Os locais e procedimentos para instalação das armadilhas e datas de coleta semanais estão descritos em MARINONI \& DUTRA (1993). A primeira coleta deuse em 04 de agosto de1986 e a última em, 27 de julho de 1987 (total de 52 semanas). A identificação das espécies de Muscidae foi feita com auxílio de chaves de identificação, descrições/ redescrições de diversos autores que trabalharam com a fauna Neotropical (CARVAlHo \& Couri 2002), e confirmada por Márcia S.Couri e um dos autores (CJBC).

Análise dos dados. O material amostrado foi analisado através de dados agrupados por semana e por mês. Para algumas análises foi utilizado o valor de captura média mensal (número de indivíduos capturados no mês dividido pelo número de semanas efetivas de captura no mês).Os dados de captura de indivíduos foram comparados aos dados meteorológicos (temperatura máxima e mínima, umidade relativa do ar e precipitação), utilizando Coeficiente de Correlação Linear, para identificar a possível influência destas variáveis na captura de indivíduos em cada uma das localidades. Para estes cálculos e elaboração dos gráficos foi empregado o programa Excel 2000 da Microsoft. Os cálculos para estimativa da riqueza de espécies foram feitos pelo programa "Estimates version 6.0b1" (CoLwELL 2001). Para análise da diversidade de Muscidae, além da riqueza de espécies (= variedade $=\mathrm{S}$ ) e do número de indivíduos $(\mathrm{N})$, foram utilizados índices ecológicos (MAGURRAN 1988; MARINONI \& DUtRa 1996; Marinoni et al. 1997). Foram aplicados os índices de diversidade de Shannon $\left(=\mathrm{H}^{\prime}\right)$ e de Brillouin $(=\mathrm{HB})$; de dominância de Berger \& Parker (= BP); de uniformidade de Shannon $(=$ H'E) e de Berger \& Parker (= UBP). Para o cálculo do logaritmo (Base 10) do fatorial necessário para obtenção do índice de diversidade de Brillouin foi utilizado o método descrito por Dutra (1995).

Material-testemunha. O material encontra-se depositado na Coleção de Entomologia Pe. Jesus Santiago Moure, do Departamento de Zoologia da Universidade Federal do Paraná.

\section{RESULTADOSEDISCUSSÃO}

Número de indivíduos (Fig. 2 ; Tabelas I, II, VI). Foram coletados 7.014 indivíduos. A maior abundância ocorreu em Ponta Grossa (3.559 indivíduos, correspondendo a $1,76 \%$ do total de 202.187 dípteros), seguida de Guarapuava (2.001 indivíduos, $4,45 \%$ do total de 44.492 ) e Colombo (1.454 indivíduos, 7,97\% do total de 18.248).

Em Ponta Grossa, a maior média de captura semanal $(71,20$ indivíduos) foi em outubro; em Guarapuava, a maior média $(39,40)$ foi em agosto; e Colombo $(28,95)$ em outubro (Tabela II). Os dados de captura de Muscidae, em Ponta Grossa e Colombo, indicam uma sazonalidade que corresponde àquela observada para os insetos em geral coletados pelo PROFAUPAR (MARINONI \& DUTRA 1993), nas áreas mais frias (com mais de cinco geadas anuais) e de mais altas latitudes e altitudes. Nestes locais, houve uma maior captura nos meses de primavera e/ou verão, com temperaturas mais quentes e dias mais longos. Em Guarapuava, no entanto, incluída entre as localidades de clima mais frio, a maior captura deu-se no mês de agosto, com declínio continuado pelos meses de primaveraverão até os meses de outono-inverno. O mês excepcional de

Tabela I. Número total de exemplares de Muscidae, por localidade, capturados durante 12 meses, de 04 de agosto de 1986 a 27 de julho de 1987.

\begin{tabular}{lrrrrrrrrrrrrr}
\hline Localidade & Ago & Set & Out & Nov & Dez & Jan & Fev & Mar & Abr & Mai & Jun & Jul & Total \\
\hline Colombo & 206 & 173 & 261 & 239 & 92 & 138 & 97 & 39 & 7 & 5 & 32 & 165 & 1454 \\
Ponta Grossa & 203 & 335 & 839 & 728 & 255 & 418 & 157 & 197 & 95 & 68 & 82 & 182 & 3559 \\
Guarapuava & 718 & 473 & 343 & 225 & 32 & 42 & 24 & 32 & 22 & 10 & 17 & 63 & 2001 \\
\hline Total & 1127 & 981 & 1443 & 1192 & 379 & 598 & 278 & 268 & 124 & 83 & 131 & 410 & 7014 \\
\hline
\end{tabular}


Tabela II. Captura média de Muscidae (número de exemplares/número de amostras no mês), por localidade, capturados durante 12 meses, de 04 de agosto de 1986 a 27 de julho de 1987.

\begin{tabular}{|c|c|c|c|c|c|c|c|c|c|c|c|c|c|}
\hline Localidade & Ago & Set & Out & Nov & Dez & Jan & $\mathrm{Fev}$ & Mar & Abr & Mai & Jun & Jul & Total \\
\hline Colombo & 51,5 & 34,6 & 65,3 & 59,8 & 18,4 & 34,5 & 24,3 & 7,8 & 1,8 & 1,7 & 6,4 & 41,3 & 28,95 \\
\hline Ponta Grossa & 50,8 & 67,0 & 209,8 & 182,0 & 51,0 & 104,5 & 39,3 & 39,4 & 31,7 & 17,0 & 16,4 & 45,5 & 71,20 \\
\hline Guarapuava & 179,5 & 94,6 & 85,8 & 56,3 & 6,4 & 10,5 & 6,0 & 6,4 & 5,5 & 2,5 & 3,4 & 15,8 & 39,40 \\
\hline
\end{tabular}

captura média, em agosto, com 179 indivíduos, resultou em uma linha de tendência (polinomial de $3^{\text {a }}$ ordem) de captura totalmente distinta das observadas em Colombo e Ponta Grossa (Fig. 2). Apesar de não ser possível relacionar simplesmente o alto número de exemplares capturados em agosto, em Guarapuava, com os valores de umidade relativa do ar observados, deve ser destacado que os mais altos coeficientes de correlação da captura média das três localidades foram com os valores de umidade relativa do ar (Tabela VI). Em períodos mais secos houve uma tendência para maior captura de indivíduos, nas três localidades, com a linha de tendência (polinomial de $3^{\mathrm{a}}$ ordem) da umidade relativa do ar apresentandose como espelho da linha de tendência da captura média. Para as três localidades, o coeficiente de correlação entre a captura média e a umidade relativa (Tabela VI) foi negativo, o inverso dos valores do parâmetro, indicando uma maior intensidade de vôo dos muscídeos nos períodos mais secos.

Em estudos de comportamento e de sazonalidade de Syrphidae (Diptera) também foi observada uma diminuição da atividade de vôo quando a umidade é mais alta (GILBERT 1985; Maier \& Waldbauer 1979; Marinoni \& Bonatto 2002). MARINONI \& BonATTO (2002), também com material proveniente do PROFAUPAR, estudaram a sazonalidade de três espécies de Syrphidae, e anotaram que dentre as duas presentes em Guarapuava, a espécie mais abundante, Toxomerus tibicen (Wiedemann, 1830) teve o maior número de indivíduos capturados em agosto, como ocorreu com Muscidae. E, da mesma forma que no presente estudo, verifica-se que a abundância decresceu para os meses de primavera-verão.

Mesmo associando a queda do número de exemplares capturados à maior umidade relativa do ar, observa-se forte redução da captura média em dezembro, nas três localidades (Fig. 2; Tabela II). Este fenômeno deve ter decorrido não apenas pelo volume de precipitação de chuva, quase o dobro do volume de novembro, mas também pelo número de dias chuvosos, que foi superior ao do mês de novembro e dos meses de verão. Em Colombo, em dezembro houve 24 dias com chuva / 7 dias sem chuva; em novembro 13 / 17. Em Guarapuava, em dezembro, 20 dias com chuva / 11 sem chuva, em novembro 13/18. Em Ponta Grossa, em dezembro, 19 dias com chuva / 12 dias sem chuva; em novembro, 12 / 18.

Número de espécies (Fig. 1; Tabelas IV, V). Foram identificadas 91 espécies. Ponta Grossa foi o local que apresentou maior riqueza, com 77 espécies, seguido de Guarapuava, com 71 e Colombo com 65 (Tabela III). Os mais altos valores de captura mensal de espécies foram em outubro e novembro em Ponta Grossa, com 53 espécies; em setembro em Guarapuava, com 52; e em outubro em Colombo, com 37 espécies (Tabela IV).

O número acumulado de espécies capturadas mensalmente (Fig 1; Tabela V), mostra que, no terceiro mês de coleta, em Guarapuava, já se somavam $90 \%$ do total de espécies capturadas (64/71); em Ponta Grossa, 86\% (66/77); e 69\% (46/65) em Colombo. Nas três localidades reunidas, nos três primeiros meses foram capturadas 81 , correspondendo a $89 \%$ do total. Este percentual de captura média foi superior aos encontrados para Ctenuchidae, Saturniidae e Sphingidae, com 66\%, 65\% e $71 \%$, respectivamente, dentro dos estudos do PROFAUPAR (MARINONi \& Dutra 1996; MARINONi et al. 1997; MARINONI et al.1999).

Estimativa da riqueza de espécies de Muscidae (Tabela VIII). Foram obtidos diferentes valores de riqueza de espécies através do cálculo de vários estimadores (Estimates version 6.0b1),

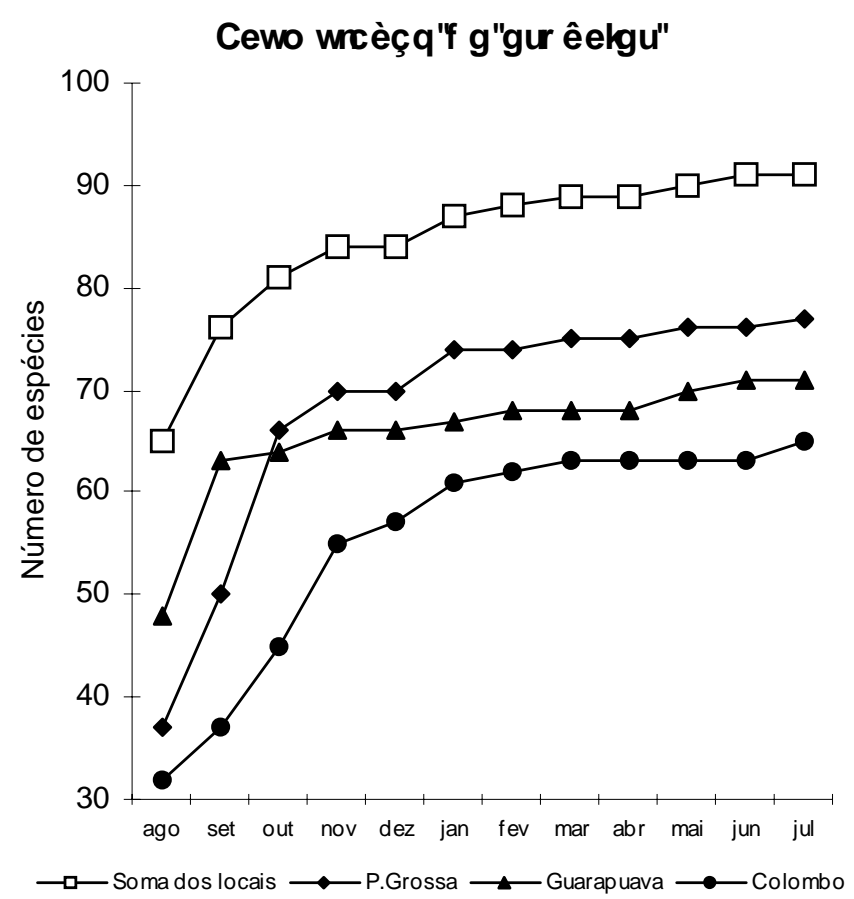

Fig. 1. Número acumulado de espécies de Muscidae capturadas mensalmente nas três localidades estudadas, durante o período de 04 de agosto de 1986 a 27 de julho de 1987. 

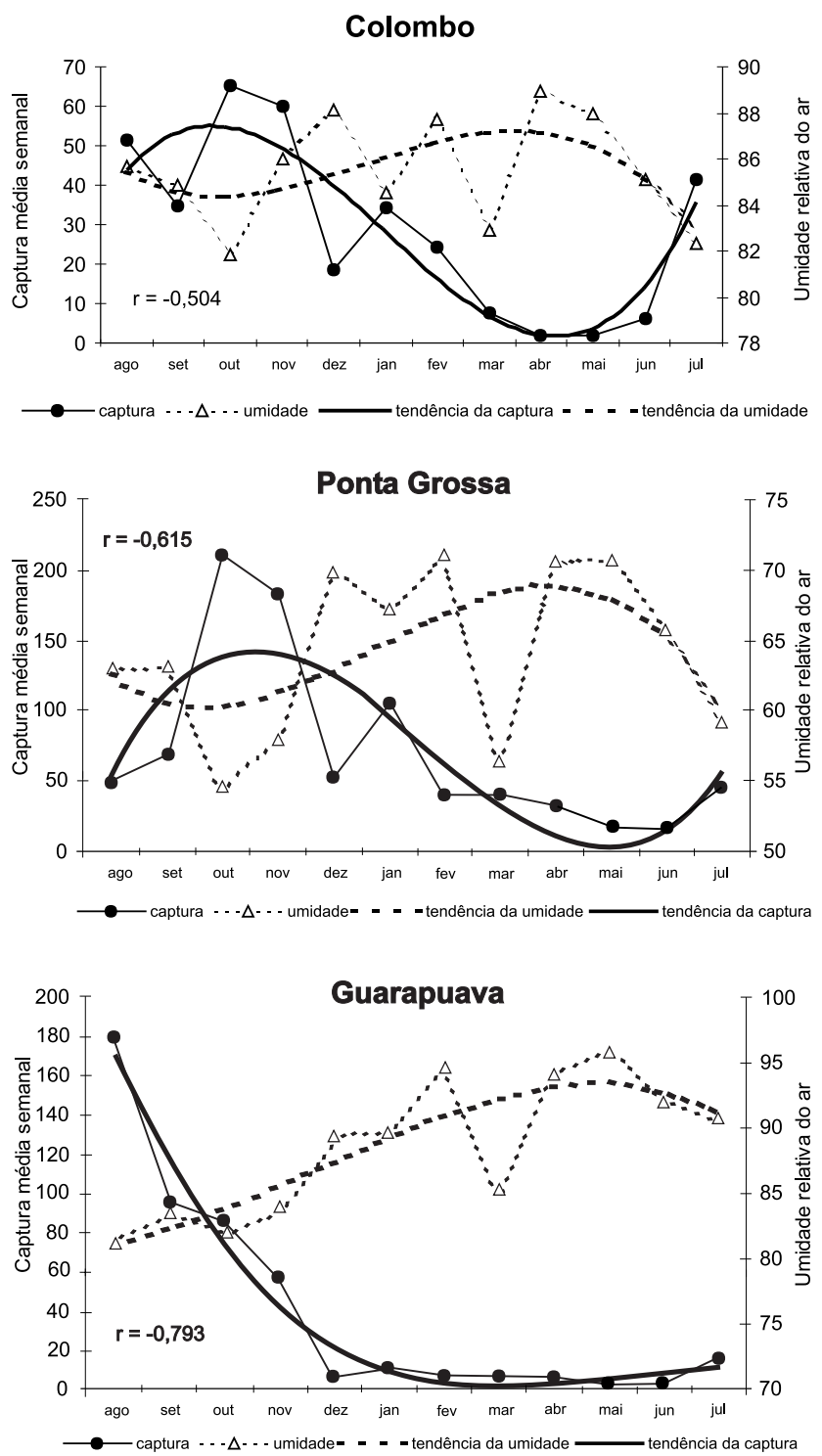

Fig. 2. Gráfico de cada uma das localidades estudadas, com o valor da captura média mensal (círculo preto), com curva de tendência (linha cheia); e os dados da umidade relativa do ar (triângulo vazado), com a curva de tendência (linha tracejada), durante o período de 04 de agosto de 1986 a 27 de julho de 1987. Linhas de tendência definidas por polinômio de $3^{\mathrm{a}}$ ordem.

utilizando dados semanais de captura. Em Colombo, onde foram capturadas 65 espécies, a estimativa que indicou o mais alto número de espécies a ser ainda possivelmente capturada foi o método de jackknife1, com 73 ( \pm 2 ) espécies (mais 12\%); e a estimativa com mais baixo valor foi indicada pelo cálculo de Chao1, com 66 ( \pm 2 ) espécies. Em Ponta Grossa, foram capturadas 77 espécies, e a estimativa mais alta foi dada pelo método de jackknife2, com 88 espécies (mais 14\%) e a mais baixa pelo método de Bootstrap, com 80 espécies. Em Guarapuava, com 71 espécies capturadas, a maior estimativa para a riqueza foi de 85 espécies pelo método de jackknife2, e a menor, com 75 espécies, a que representa a assíntota da curva de MichaelisMenten. Em Guarapuava observaram-se os maiores valores percentuais da riqueza de espécies estimada comparada aos valores de espécies efetivamente capturadas, com cerca de $20 \%$ acima para o método de jackknife2 e de cerca de $6 \%$ para a assíntota de Michaelis-Mentem.

Diversidade de Muscidae (Tabela VII). Considerando-se os dados de riqueza de espécie e de abundância, Ponta Grossa com 77 espécies e 3.559 indivíduos capturados, apresentou a maior diversidade. Corroborando esta interpetação, os índices de Shannon (H') e de Brillouin (HB) também indicam Ponta Grossa como o local de maior diversidade. As três localidades têm índices de diversidade muito semelhantes. As diferenças maiores são observadas na uniformidade que, pelo índice de Berger \& Parker, indica Colombo como sendo o local onde há menor dominância das espécies mais abundantes.

Os diferentes valores de riqueza de espécies e dos índices de diversidade, encontrados para Muscidae nos três locais, concordam proporcionalmente com os valores encontrados para as famílias de Lepidoptera, Ctenuchidae, Saturniidae e Sphingidae (MARINONI \& Dutra 1996; MARINONI et al. 1997; MARINONi et al. 1999). Ponta Grossa apresenta-se como a localidade mais rica em espécies, e Colombo a mais pobre; o mesmo ocorre quanto à diversidade, maior em Ponta Grossa e menor em Colombo. Quanto à uniformidade, no entanto, apesar de terem sido observados valores de dominância mais baixos em Ponta Grossa, com relação às famílias de Lepidoptera, não o foi com relação a Muscidae. Nesta, os dados indicam uma menor dominância em Colombo, seguida de Guarapuava.

As três áreas foram florísticamente alteradas pela ação do homem, segundo as observações de Hatschbach (in MARINONI $\&$ DUTRA 1993), porém Colombo foi a que se apresentou mais destruída, com Ponta Grossa e Guarapuava apresentando melhores condições de regeneração. Esta situação é corroborada pelos dados da fauna das famílias de Lepidoptera já estudadas, que apontam Ponta Grossa como a localidade mais rica em espécies e com maior abundância de indivíduos, e Colombo como a mais pobre e com menor abundância.

Faunística. Ocorrência, abundância e alguns dados sazonais de espécies (Tabelas III, IX). Das 91 espécies de Muscidae, 52 (57\%) são comuns às três localidades. Ponta Grossa e Guarapuava têm 61 espécies em comum; Colombo e Ponta Grossa, 59 espécies; Colombo e Guarapuava, 54 espécies. O maior número de espécies comuns a Ponta Grossa e Guarapuava (61) e Ponta Grossa e Colombo (59), e o menor entre Guarapuava e Colombo (54), é coerente com a posição geográfica de Ponta Grossa, intermediária entre as outras duas. Quanto às espécies exclusivas, nove ocorreram em Ponta Grossa, oito em Guarapuava e quatro em Colombo (Tabela III).

A soma dos percentuais das cinco espécies dominantes de cada local foram similares, em torno de 50\%. Dentre estas espécies, com exceção de Stomopogon sp.1 que não foi encontrada em Guarapuava, a maioria delas ocorreu nas três localidades (Tabela IX). 
Tabela III. Lista das espécies capturadas em três localidades do Estado do Paraná, durante o período de 04 de agosto de 1986 a 27 de julho de 1987. (CO) Colombo; (PG) Ponta Grossa; (GUA) Guarapuava; (*) espécies com primeiro registro de ocorrência no Estado do Paraná.

\begin{tabular}{|c|c|c|c|c|}
\hline Espécies & $\mathrm{CO}$ & PG & GUA & Total \\
\hline Bithoracochaeta atricornis Malloch, 1934 & 7 & 34 & 19 & 60 \\
\hline Bithoracochaeta leucoprocta (Wiedemann, 1830) & 0 & 1 & 0 & 1 \\
\hline Bithoracochaeta plumata Albuquerque, 1955 * & 0 & 0 & 2 & 2 \\
\hline Cariocamyia maculosa Snyder,1951 & 0 & 0 & 2 & 2 \\
\hline Coenosia sp.1 & 5 & 0 & 0 & 5 \\
\hline Coenosia sp.2 & 4 & 33 & 28 & 65 \\
\hline Coenosia sp.3 & 0 & 1 & 0 & 1 \\
\hline Coenosia sp.4 & 0 & 1 & 0 & 1 \\
\hline Cordiluroides megalopyga Albuquerque, 1954 & 0 & 0 & 6 & 6 \\
\hline Cyrtoneuropsis incognita (Snyder, 1954)* & 117 & 423 & 56 & 596 \\
\hline Cyrtoneuropsis mellina (Stein, 1918) * & 23 & 98 & 21 & 142 \\
\hline Cyrtoneuropsis sp.1 & 11 & 37 & 3 & 51 \\
\hline Dolichophaonia catharinensis Carvalho,1983 & 4 & 3 & 3 & 10 \\
\hline Dolichophaonia giacomeli Carvalho,1981 & 0 & 0 & 1 & 1 \\
\hline Dolichophaonia plaumanni Carvalho, 1983 & 5 & 5 & 7 & 17 \\
\hline Dolichophaonia santoamarensis Albuquerque, 1958 & 0 & 0 & 1 & 1 \\
\hline Dolichophaonia sp.1 & 2 & 0 & 1 & 3 \\
\hline Dolichophaonia sp.2 & 0 & 0 & 1 & 1 \\
\hline Dolichophaonia sp.3 & 0 & 0 & 1 & 1 \\
\hline Helina praecipua (Walker, 1853) & 26 & 22 & 40 & 88 \\
\hline Helina $\mathbf{s p} .1$ & 0 & 1 & 11 & 12 \\
\hline Helina sp. 2 & 17 & 15 & 26 & 58 \\
\hline Helina sp.3 & 0 & 9 & 10 & 19 \\
\hline Helina sp.4 & 1 & 0 & 0 & 1 \\
\hline Morellia dendropanacis Pamplona \& Couri, $1995 *$ & 1 & 17 & 5 & 23 \\
\hline Morellia humeralis (Stein,1918) & 0 & 3 & 1 & 4 \\
\hline Morellia maculipennis Macquart, 1846 & 0 & 13 & 1 & 14 \\
\hline Morellia violacea (Robineau-Desvoidy,1830) & 5 & 5 & 1 & 11 \\
\hline Morellia xanthoptera Pamplona, $1986 *$ & 0 & 10 & 0 & 10 \\
\hline Mydaea plaumanni Snyder,1941 & 34 & 69 & 28 & 131 \\
\hline Myospila obscura Shannon \& Del Ponte, 1926 * & 3 & 53 & 8 & 64 \\
\hline Neodexiopsis emmesa (Malloch, 1934) & 2 & 5 & 3 & 10 \\
\hline Neodexiopsis flavipalpis Albuquerque, 1956 & 34 & 672 & 332 & 1038 \\
\hline Neodexiopsis neoaustralis Snyder,1957 * & 1 & 13 & 4 & 18 \\
\hline Neodexiopsis nigerrima (Malloch,1934) & 7 & 338 & 23 & 368 \\
\hline Neodexiopsis setipuncta Snyder, 1957 & 15 & 57 & 177 & 249 \\
\hline Neodexiopsis vulgaris Couri \& Albuquerque, 1979 & 172 & 30 & 76 & 278 \\
\hline Neodexiopsis $\mathbf{s p} .1$ & 2 & 5 & 5 & 12 \\
\hline Neodexiopsis $\mathbf{s p} .2$ & 0 & 16 & 1 & 17 \\
\hline Neodexiopsis sp. 3 & 5 & 6 & 4 & 15 \\
\hline Neodexiopsis $\mathbf{s p} .4$ & 9 & 56 & 49 & 114 \\
\hline Neodexiopsis sp.5 & 38 & 87 & 52 & 177 \\
\hline Neodexiopsis sp.6 & 27 & 84 & 31 & 142 \\
\hline Neodexiopsis sp.7 & 6 & 0 & 10 & 16 \\
\hline Neodexiopsis $\mathbf{s p} .8$ & 9 & 0 & 0 & 9 \\
\hline Neodexiopsis sp.9 & 4 & 8 & 22 & 34 \\
\hline Neodexiopsis sp. 10 & 16 & 48 & 72 & 136 \\
\hline Neodexiopsis sp.11 & 2 & 118 & 218 & 338 \\
\hline Neomuscina atincticosta (Snyder, 1949) & 3 & 1 & 3 & 7 \\
\hline Neomuscina inflexa (Stein, 1918)* & 38 & 48 & 5 & 91 \\
\hline Neomuscina pictipennis pictipennis Bigot, $1875 *$ & 1 & 7 & 0 & 8 \\
\hline
\end{tabular}


Tabela III. (continuação)

\begin{tabular}{|c|c|c|c|c|}
\hline Espécies & $\mathrm{CO}$ & PG & GUA & Total \\
\hline Neomuscina shadei Snyder, 1949 & 7 & 16 & 7 & 30 \\
\hline Neomuscina tinctinervis (Stein, 1918) * & 3 & 2 & 6 & 11 \\
\hline Neomuscina zosteris Shannon \& Del Ponte,1926 & 133 & 131 & 13 & 277 \\
\hline Neomuscina sp.1 & 2 & 15 & 0 & 17 \\
\hline Neomuscina $\mathbf{s p . 2}$ & 9 & 12 & 3 & 24 \\
\hline Neomuscina sp.3 & 19 & 0 & 0 & 19 \\
\hline Neomuscina sp.4 & 7 & 13 & 0 & 20 \\
\hline Neurotrixa $\mathbf{s p} .1$ & 35 & 14 & 5 & 54 \\
\hline Neurotrixa sp.2 & 24 & 7 & 4 & 35 \\
\hline Phaonia advena Snyder, 1957 & 22 & 7 & 6 & 35 \\
\hline Phaonia annulata (Albuquerque, 1957) & 1 & 22 & 24 & 47 \\
\hline Phaonia bigoti (Albuquerque, 1957) & 11 & 58 & 0 & 69 \\
\hline Phaonia grajauensis (Albuquerque, 1957) & 2 & 63 & 89 & 154 \\
\hline Phaonia lentiginosa (Snyder, 1957) & 13 & 10 & 3 & 26 \\
\hline Phaonia nigriventris (Albuquerque, 1954) & 1 & 2 & 29 & 32 \\
\hline Phaonia praesuturalis (Stein, 1904) & 0 & 8 & 0 & 8 \\
\hline Phaonia shannoni Carvalho \& Pont, 1993 & 2 & 42 & 30 & 74 \\
\hline Phaonia trispila (Bigot, 1885) & 82 & 97 & 41 & 220 \\
\hline Phaonia sp.1 & 2 & 3 & 2 & 7 \\
\hline Phaonia sp.2 & 3 & 5 & 2 & 10 \\
\hline Phaonia sp.3 & 17 & 2 & 1 & 20 \\
\hline Phaonia sp.4 & 0 & 24 & 20 & 44 \\
\hline Phaonia sp.5 & 0 & 10 & 29 & 39 \\
\hline Philornis masoni Couri, 1986 & 0 & 1 & 0 & 1 \\
\hline Pilispina pilitibia Albuquerque, $1954 *$ & 169 & 23 & 185 & 377 \\
\hline Plumispina similis Costacurta \& Carvalho, 2003* & 3 & 23 & 37 & 63 \\
\hline Polietina bicolor Albuquerque, $1956 *$ & 0 & 17 & 3 & 20 \\
\hline Polietina orbitalis (Stein,1904) & 51 & 289 & 60 & 400 \\
\hline Polietina steini (Enderlein, 1927) * & 0 & 3 & 0 & 3 \\
\hline Polietina univittata Couri \& Carvalho, 1995 & 2 & 52 & 2 & 56 \\
\hline Pseudoptilolepis fulvapoda Snyder, 1949 & 39 & 47 & 6 & 92 \\
\hline Psilochaeta pampiana Shannon \& Del Ponte, 1926 & 2 & 1 & 0 & 3 \\
\hline Stomopogon sp.1 & 129 & 47 & 0 & 176 \\
\hline Stomopogon sp.2 & 0 & 5 & 0 & 5 \\
\hline Xenomorellia sp. 1 & 5 & 15 & 1 & 21 \\
\hline Xenomorellia $\mathbf{s p . 2}$ & 0 & 2 & 0 & 2 \\
\hline Xenomorellia sp.3 & 1 & 5 & 0 & 6 \\
\hline Genero Novo A, espécie 1 & 0 & 4 & 7 & 11 \\
\hline Genero Novo B, espécie 1 & 2 & 10 & 8 & 20 \\
\hline Genero Novo C, espécie 1 & 0 & 0 & 8 & 8 \\
\hline Total de exemplares & 1454 & 3559 & 2001 & 7014 \\
\hline Total de espécies por local & 65 & 77 & 71 & 91 \\
\hline Total de espécies capturadas apenas no local & 4 & 9 & 8 & \\
\hline
\end{tabular}

A espécie com maior número de exemplares foi Neodexiopsis flavipalpis Albuquerque, 1956, tendo sido a espécie mais abundante em Ponta Grossa (672) e em Guarapuava (332) e Neodexiopsis vulgaris Couri \& Albuquerque, 1979, foi a espécie mais abundante em Colombo (172).

Em Ponta Grossa, Neodexiopsis flavipalpis foi capturada em nove dos 12 meses de amostragem, com picos de coleta em outubro (290) e novembro (262) dos 672 indivíduos capturados. Cyrtoneuropsis incognita (Snyder, 1954) foi a segunda espécie com maior número de indivíduos nesta localidade; dos 423 indivíduos, 81 foram capturados em setembro e 84 em outubro.

Em Guarapuava, Neodexiopsis flavipalpis foi capturada em seis dos 12 meses amostrados; dos 332 indivíduos, 129 foram

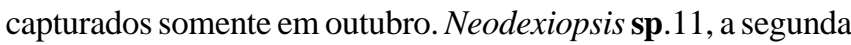


Tabela IV. Número de espécies de Muscidae, por localidade, capturadas durante 12 meses, de 04 de agosto de 1986 a 27 de julho de 1987.

\begin{tabular}{lrrrrrrrrrrrr}
\hline Localidade & Ago & Set & Out & Nov & Dez & Jan & Fev & Mar & Abr & Mai & Jun & Jul \\
\hline Colombo & 33 & 30 & 37 & 33 & 13 & 28 & 22 & 16 & 5 & 5 & 14 & 24 \\
Ponta Grossa & 38 & 40 & 53 & 53 & 22 & 47 & 37 & 40 & 26 & 22 & 28 & 34 \\
Guarapuava & 48 & 52 & 41 & 39 & 10 & 19 & 17 & 15 & 14 & 9 & 6 & 17 \\
\hline
\end{tabular}

Tabela V. Número acumulado de espécies capturadas em cada localidade, durante o período de 04 de agosto de 1986 a 27 de julho de 1987.

\begin{tabular}{lrrrrrrrrrrrr}
\hline Localidade & Ago & Set & Out & Nov & Dez & Jan & Fev & Mar & Abr & Mai & Jun & Jul \\
\hline Colombo & 33 & 38 & 46 & 56 & 57 & 61 & 62 & 63 & 63 & 63 & 63 & 65 \\
Ponta Grossa & 38 & 51 & 66 & 70 & 70 & 74 & 74 & 75 & 75 & 76 & 77 & 77 \\
Guarapuava & 48 & 63 & 64 & 66 & 66 & 67 & 68 & 68 & 68 & 70 & 71 & 71 \\
Total & 65 & 76 & 81 & 84 & 84 & 87 & 88 & 89 & 89 & 90 & 91 & 91 \\
\hline
\end{tabular}

Tabela VI. Coeficientes de correlação linear (r) entre os dados de captura média de Muscidae e as variáveis meteorológicas, por localidade. Temp.= temperatura.

\begin{tabular}{lcccc}
\hline Localidade & Temp. máxima & Temp. mínima & Umidade relativa & Pluviometria \\
\hline Colombo & 0,151 & $-0,029$ & $-0,505$ & $-0,261$ \\
Ponta Grossa & 0,420 & 0,299 & $-0,615$ & $-0,201$ \\
Guarapuava & $-0,133$ & 0,015 & $-0,793$ & $-0,293$ \\
\hline
\end{tabular}

Tabela VII. Diversidade e Uniformidade de Muscidae. (S) Número de espécies; (N) número de exemplares coletados; (HB) índice de diversidade de Brillouin; (H') índice de diversidade de Shannon; (H'E) uniformidade de Shannon; (UBP) índice de uniformidade de Berger \& Parker; (BP) índice de dominância de Berger \& Parker.

\begin{tabular}{lccccccc}
\hline Localidade & $\mathrm{S}$ & $\mathrm{N}$ & $\mathrm{HB}$ & $\mathrm{H}^{\prime}$ & $\mathrm{HE}$ & $\mathrm{UBP}$ & $\mathrm{BP}$ \\
\hline Colombo & 65 & 1454 & 1,376 & 3,196 & 0,766 & 8,453 & 0,118 \\
Ponta Grossa & 77 & 3559 & 1,392 & 3,236 & 0,745 & 5,296 & 0,189 \\
Guarapuava & 71 & 2001 & 1,386 & 3,195 & 0,750 & 6,027 & 0,166 \\
\hline
\end{tabular}

Tabela VIII. Riqueza de espécies, segundo vários estimadores.

\begin{tabular}{lccc}
\hline & Colombo & Ponta Grossa & Guarapuava \\
\hline Espécies capturadas & 65 & 77 & 71 \\
Michaelis-Menten & 72 & 81 & 75 \\
Bootstrap & 70 & 80 & 76 \\
Jack-Knife1 & $73 \pm 2$ & $84 \pm 2$ & $81 \pm 3$ \\
Jack-Knife2 & 71 & 88 & 85 \\
Chao1 & $66 \pm 2$ & $81 \pm 6$ & $80 \pm 9$ \\
Chao2 & $67 \pm 3$ & $82 \pm 7$ & $77 \pm 6$ \\
\hline
\end{tabular}


Tabela IX. Espécies com maior número de exemplares capturados nas três localidades, com percentual em relação ao total capturado em cada localidade. Localidades em ordem decrescente da porcentagem da espécie mais abundante.

\begin{tabular}{|c|c|c|c|c|c|c|}
\hline Localidade & $\begin{array}{c}\text { Total de } \\
\text { exemplares }\end{array}$ & $\begin{array}{l}\text { Total de } \\
\text { espécies }\end{array}$ & Espécies & $\begin{array}{l}\text { Número de } \\
\text { exemplares }\end{array}$ & \multicolumn{2}{|c|}{ Porcentagem } \\
\hline \multirow[t]{5}{*}{ Ponta Grossa } & \multirow{5}{*}{3559} & \multirow{5}{*}{77} & Neodexiopsis flavipalpis & 672 & 18,9 & \\
\hline & & & Cyrtoneuropsis incognita & 423 & 11,9 & \\
\hline & & & Neodexiopsis nigerrima & 338 & 9,5 & \\
\hline & & & Polietina orbitalis & 289 & 9,1 & \\
\hline & & & Neomuscina zosteris & 131 & 3,7 & 52,1 \\
\hline \multirow[t]{5}{*}{ Guarapuava } & \multirow{5}{*}{2001} & \multirow{5}{*}{71} & Neodexiopsis flavipalpis & 332 & 16,6 & \\
\hline & & & Neodexiopsis sp.11 & 218 & 10,9 & \\
\hline & & & Pilispina pilitibia & 185 & 9,3 & \\
\hline & & & Neodexiopsis setipuncta & 177 & 8,9 & \\
\hline & & & Phaonia grajauensis & 89 & 4,5 & 50,2 \\
\hline \multirow[t]{5}{*}{ Colombo } & \multirow{5}{*}{1454} & \multirow{5}{*}{65} & Neodexiopsis vulgaris & 172 & 11,8 & \\
\hline & & & Pilispina pilitibia & 169 & 11,6 & \\
\hline & & & Neomuscina zosteris & 133 & 9,2 & \\
\hline & & & Stomopogon sp.1 & 129 & 8,9 & \\
\hline & & & Cyrtoneuropsis incognita & 117 & 8,1 & 49,6 \\
\hline
\end{tabular}

espécie mais abundante nesta localidade, teve 114 indivíduos capturados em agosto, de um total de 218.

Em Colombo, Neodexiopsis vulgaris foi capturada em 11 dos 12 meses amostrados; teve 33 indivíduos capturados em cada um dos meses de outubro e novembro; Pilispina pilitibia Albuquerque, 1954 (169) foi capturada em oito dos 12 meses amostrados, tendo os meses de outubro (44) e novembro (46) como os de maior abundância.

Baseada na dominância das espécies, comparando-se as estruturas das comunidades utilizando coeficiente de correlação linear, Ponta Grossa e Guarapuava são mais assemelhadas ( $\mathrm{r}=$ $0,994)$, enquanto Colombo é mais assemelhada a Guarapuava ( $\mathrm{r}$ $=0,962$ ).

Muscidae do PROFAUPAR x Muscidae até então conhecidos no Paraná. A partir do presente estudo, o conhecimento da fauna de Muscidae do Estado do Paraná em relação à fauna Neotropical, passou a aproximadamente 11,55\% para as espécies e 39,08\% para os gêneros.

Não havia registro de ocorrência no Brasil, de acordo com CARVALHO \& et al. (1993), para o gênero Stomopogon Malloch, 1930 (Peru, Bolivia, Chile, Argentina) e para as seguintes espécies: Neodexiopsis emmesa (Malloch, 1934) (Costa Rica, Panamá, Venezuela), Neodexiopsis nigerrima (Malloch, 1934) (Chile, Argentina), Neodexiopsis setipuncta Snyder, 1957 (Argentina), Neomuscina shadei Snyder, 1949 (Paraguai), Philornis masoni Couri, 1986 (Uruguai).

Além das espécies citadas acima, foram encontradas 14 espécies que não tinham registro de ocorrência no Estado do Paraná, conforme assinalado na Tabela III.

\section{OBSERVAÇÕES FINAIS}

Baseando-se na fauna de Muscidae, coletada através do uso de armadilha Malaise, durante o período de 4 de agosto de 1986 a 27 de julho de 1987, em três localidades do Estado do Paraná, observa-se que:

1. Foram capturados 7.014 muscídeos de 91 espécies; a maior riqueza de espécies e abundância de indivíduos foi em Ponta Grossa (77 espécies e 3559 indivíduos).

2. A espécie com maior número de exemplares foi Neodexiopsis flavipalpis, tendo sido a espécie mais abundante em Ponta Grossa (672) e em Guarapuava (332) e Neodexiopsis vulgaris foi a espécie mais abundante em Colombo (172).

3. A sazonalidade de Muscidae, em Ponta Grossa e Colombo, é semelhante àquela observada para a maioria dos insetos da mesma região, ou seja, maior número de espécies e maior abundância de indivíduos nos meses de primavera-verão; em Guarapuava houve um comportamento diferenciado, com maior abundância no fim do inverno (agosto-setembro).

4. Há uma tendência para uma captura maior de indivíduos nos períodos de mais baixa umidade relativa do ar.

5. Nos três primeiros meses de coleta (agosto-outubro) foram coletados cerca de 70 a $90 \%$ das espécies ( $89 \%$ na somatória dos locais); estes percentuais foram superiores aos observados em famílias de Lepidoptera (Ctenuchidae, Saturniidae e Sphingidae).

6. O número ainda possível de captura de diferentes espécies, segundo vários estimadores de riqueza de especies, não ultrapassa a $20 \%$ das espécies já capturadas.

7. Ponta Grossa é apontada como a mais rica em espécies e com maior abundância de indivíduos, e Colombo a mais pobre e com menor abundância, corroborando os dados observados para famílias de Lepidoptera (Ctenuchidae, Saturniidae e Sphingidae).

8. Cinqüenta e duas espécies (57\%) foram comuns aos três locais; Ponta Grossa e Guarapuava foram as mais assemelhadas faunísticamente, e Colombo mostrou-se mais semelhante a 
Ponta Grossa; a condição faunística de Ponta Grossa é coerente com a posição geográfica, intermediária entre as duas outras localidades. O local com maior número de espécies exclusivas foi Ponta Grossa.

Agradecimentos. À Márcia Souto Couri (Museu Nacional do Rio de Janeiro) pela identificação ou confirmação de várias espécies de Muscidae, e à Luciane Marinoni pela revisão do Abstract. Reiteramos os agradecimentos a todos aqueles que trabalharam no PROFAUPAR Levantamento da Fauna Entomológica no Estado do Paraná, nos processos de coleta e triagem preliminar do material entomológico.

\section{REFERÊNCIAS}

Carvalho, C. J. B. De \& M. S. Couri. 2002. Part II. Apical Groups. In: C. J. B. de Carvalho (ed). Muscidae (Diptera) of the Neotropical Region: Taxonomy. Curitiba, Ed. UFPR, $287 \mathrm{p}$.

Carvalho, C. J. B. de; M. S. Couri; A. C. Pont; D. M. Pamplona \& S. M. Lopes. 1993. Part II. Muscidae. In: C. J. B. de Carvalho (ed). A Catalogue of the Fanniidae and Muscidae (Diptera) of the Neotropical region. São Paulo, Sociedade Brasileira de Entomologia, $201 \mathrm{p}$.

Colwell, R. K. 2001. EstimateS: Statistical estimation of species richness and shared species from samples. Version 6.0b1. User's guide and application. (disponível em http:/viceroy.eeb.uconn.edu/ estimates6; acesso em 20/03/2002).

Costacurta, N. C.; C. J. B. de Carvalho \& M. S. Couri. 2003. A new species of Plumispina Albuquerque (Diptera, Muscidae) from Southern Brasil. Proceedings of the Entomological Society of Washington 105(1): 127-130.
Dutra, R. R. C. 1995. Um procedimento para o cálculo do Índice de diversidade de Brillouin. Revista Brasileira de Zoologia 12(4): 1025-1028.

GILbERT, F. S. 1985. Diurnal activity patterns in hoverflies (Diptera, Syrphidae). Ecolological Entomology 10: 385-392.

MagurRan, A. E. 1988. Ecological diversity and its measurement. Princeton, Princeton University Press, 179 p.

Marinoni, L. \& S. R. Bonatto. 2002. Sazonalidade de três espécies de Syrphidae (Insecta, Diptera) capturadas com armadilha Malaise no Estado do Paraná, Brasil. Revista Brasileira de Zoologia 19(1): 95-104.

Marinoni, R. C. \& R. R. C. Dutra. 1993. Levantamento da Fauna Entomológica no Estado do Paraná. I. Introdução. Situações climática e florística de oito pontos de coleta. Dados faunísticos de agosto de 1986 a julho de1987. Revista Brasileira de Zoologia 8 (1/2/3/4): 31-73 [1991].

Marinoni, R. C. \& R. R. C. Dutra. 1996. Levantamento da Fauna Entomológica no Estado do Paraná. II. Ctenuchidae (Lepidoptera). Revista Brasileira de Zoologia 13(2): 435-461.

Marinoni, R. C.; R. R. C. Dutra \& M. M. CASAgRande. 1997. Levantamento da Fauna Entomológica no Estado do Paraná. III. Saturniidae (Lepidoptera). Revista Brasileira de Zoologia 14(2): 473-495.

Marinoni, R. C.; R. R. C. Dutra \& O. H. H. MielKe. 1999. Levantamento da Fauna Entomológica no Estado do Paraná. IV. Sphingidae (Lepidoptera). Revista Brasileira de Zoologia 16(supl.2): 223240.

Maier, C. T. \& G. P. Waldbauer. 1979. Diurnal activity patterns of flower flies (Diptera: Syprhidae). Annals of the Entomological Society of America 72: 55-61.

Townes, H. 1972. A light-weight Malaise trap. Entomological News 83: 239-247.

Veloso, P. H. \& L. Góes Filho. 1982. Fitogeografia Brasileira. Classificação fisionômica-ecológica da vegetação neotropical. Boletim Técnico Projeto RADAMBRASIL, Série Vegetação, 85 p. 